\title{
EPITAXIAL GROWTHS OF II-VI COMPOUNDS ON (110) SUBSTRATES
}

\author{
G. CYwiński, T. Wojtowicz, K. Kopalko, G. Karczewski \\ AND J. KossuT
}

Institute of Physics, Polish Academy of Sciences

Al. Lotników 32/46, 02-668 Warsaw, Poland

\begin{abstract}
We studied epitaxial growth conditions of II-VI semiconductors on (110) substrates, which is indispensable for fabrication of T-shaped quantum wire structures. We experimented with different types of (110)-oriented substrates and monitored the surface quality of deposited layers in situ by reflection high energy electron diffraction and ex situ by photoluminescence. The aim of this work is to find optimum growth conditions of II-VI compounds on a cleaved edge of a superlattice as required by the overgrowth method.
\end{abstract}

PACS numbers: $68.55 .-\mathrm{a}$

The first and necessary stage of fabrication of T-shaped quantum wires [1] by an overgrowth on a cleaved edge of a superlattice involves mastering epitaxial growth on (110)-oriented substrates. The reason for this is that the overgrowth method of producing quasi one-dimensional structures requires a very high quality of the quantum wells formed on the (110) cleave. Optimization of the growth conditions of the size-quantized heterostructures on such substrates is a prerequisite for obtaining a strong photoluminescence signal characterized by a narrow line width of the emission from quantum wells on (110) cleaved edges. Such orientation of the substrates is difficult from the point of view of epitaxy [2] and, usually, the structures grown with (110) orientation are inferior in quality compared to (100)-oriented counterparts, the fact related to the weak polarity of the former. In particular, it is known that the sticking coefficient of $\mathrm{Te}_{4}$ to (110) surface is much smaller than to (100) surface. Therefore, when growing on (110) surface one is bound to strongly increase the $\mathrm{Te} / \mathrm{Cd}$ flux ratio and/or to lower the substrate temperature. Both factors lead to a worse structural quality of the grown material compared to that obtained on (100) oriented substrates.

We have tried three different types of substrates and aimed at optimization of the conditions of growth of CdTe and other II-VI compounds. To monitor the results we used in situ reflection high energy electron diffraction (RHEED) and ex situ - photoluminescence (PL). First attempts involved (110)-oriented CdTe bulk substrates. On changing the incident particle flux ratio as well as the substrate temperature it was possible to optimize the molecular beam epitaxy (MBE) of 
thick CdTe layers [3]. We found that the best growth conditions corresponded to a relatively low substrate temperature (about $45^{\circ} \mathrm{C}$ lower than for the growth of optimized (100)-oriented material) and about three times greater flux ratio of $\mathrm{Te} / \mathrm{CdTe}$ (or $\mathrm{Te} / \mathrm{Cd}$ ). Such conditions led to a growth characterized by sharp streaks in the RHEED image without appearance of spotty diffraction appearance after several minutes of the growth (Fig. 1). The growth was stable and we have not observed any evidence of its deterioration. We checked that characteristic RHEED patterns clearly revealed the symmetry of the (110) surface.

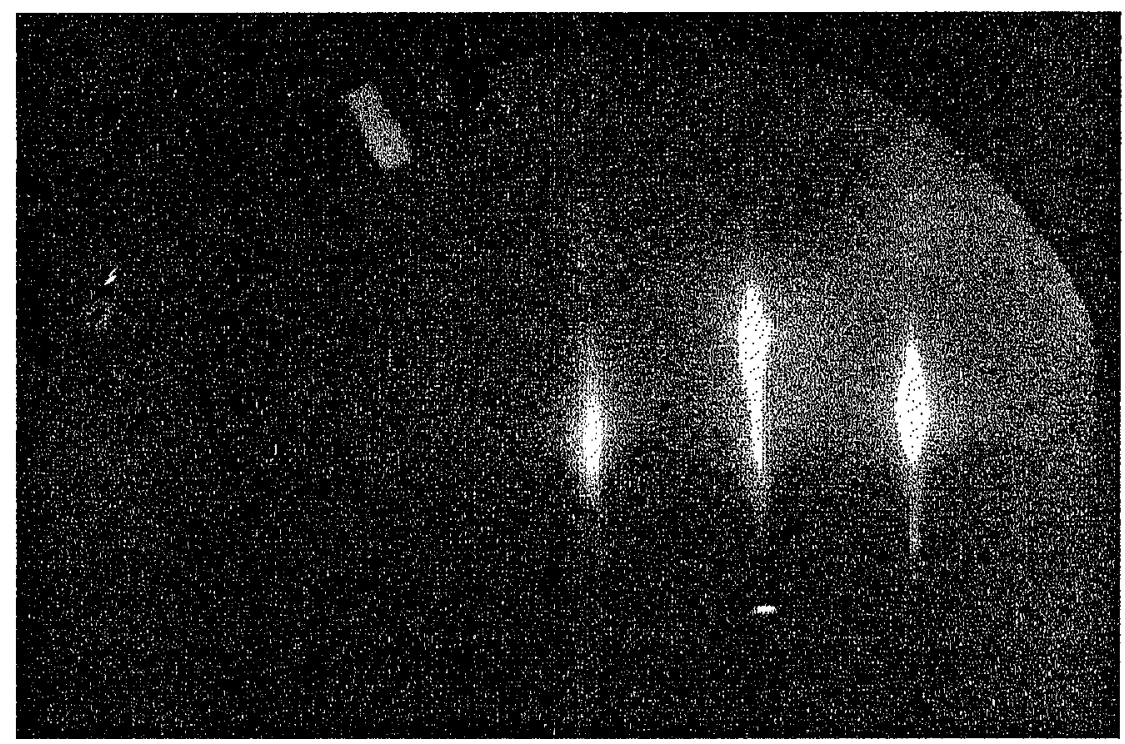

Fig. 1. RHEED image of (110) CdTe surface after MBE growth on bulk substrates CdTe.

For $e x$ situ characterization we fabricated samples containing the quantum wells made of CdTe with CdMnTe or CdMgTe barriers. When growing the II-VI ternaries on (110) CdTe bulk substrates we grew in parallel reference samples on (100)-oriented CdTe substrates. In all our cases the optical quality of (110)-oriented structures was never as good as in the case of (100)-oriented substrates. In the former case the photoluminescence signal was rather weak and the lines were wider than in the samples obtained by epitaxy along the easy-to-grow directions. However, it was difficult to correlate the growth conditions with the quality of the PL signal from the quantum wells. After a prolonged growth we observed that the RHEED images from the (110)-oriented structures became very dark with the diffraction pattern hardly noticeable. We did not observe RHEED intensity oscillations when growing (110)-CdTe. These shortages of (110)-oriented substrates of CdTe made us to look for other possibilities.

The second type of substrates used in the study was (110)-oriented GaAs. We observed that after approximately 20 minutes of the growth of $\mathrm{CdTe}$ (about 
$0.2 \mu \mathrm{m})$ the RHEED image disappeared indicating that the growing material had a low crystalline perfection. This was probably caused by a large lattice mismatch between GaAs and CdTe. We were not able to obtain good growth conditions CdTe in the case of the (110) GaAs substrates.

Finally, we have experimented with deposition of $\mathrm{CdTe}, \mathrm{CdMnTe}$, and $\mathrm{CdMgTe}$ on the cleaved edge of $10 \mu \mathrm{m} \mathrm{CdTe}$ on GaAs hybrid substrates previously grown with (100)-orientation (with (110) surface exposed by the cleave). The hybrid substrates were thinned by a mechanical method down to about $100 \mu \mathrm{m}$ and the cleave was performed in ultrahigh vacuum conditions in the MBE growth chamber. In particular, we deposited a thin CdMnTe layer and, then, three 24, 12, and $6 \mathrm{ML}$ wide quantum wells embedded in CdMnTe barriers. Unfortunately, the geometry of the arrangement did not allow us to observe RHEED images of the (110) cleaved surface. The optically active region of the structure was limited only to the stripe grown on cleaved CdTe buffer. The rest of the material deposited on cleaved GaAs did not reveal any PL signal from the QW - as checked in a microluminescence setup - probably due to a low crystalline quality of this part of the structure. The observed strong photoluminescence possessed sharp spectral lines (Fig. 2) whose widths were comparable to those from the samples grown (110) bulk substrates. Thus, we have demonstrated that in spite of the difficulties of growth on the (110) oriented substrates it is possible to obtain CdTe-based structures with a quite good optical quality. As mentioned above this experimental growth has been a prelude to producing the T-shaped wires [4]. Unfortunately, to produce T-shaped wires, one has to deposit a well layer directly on the (110)-cleaved surface without any intermediate barrier layer, i.e., to skip the first CdMnTe over-

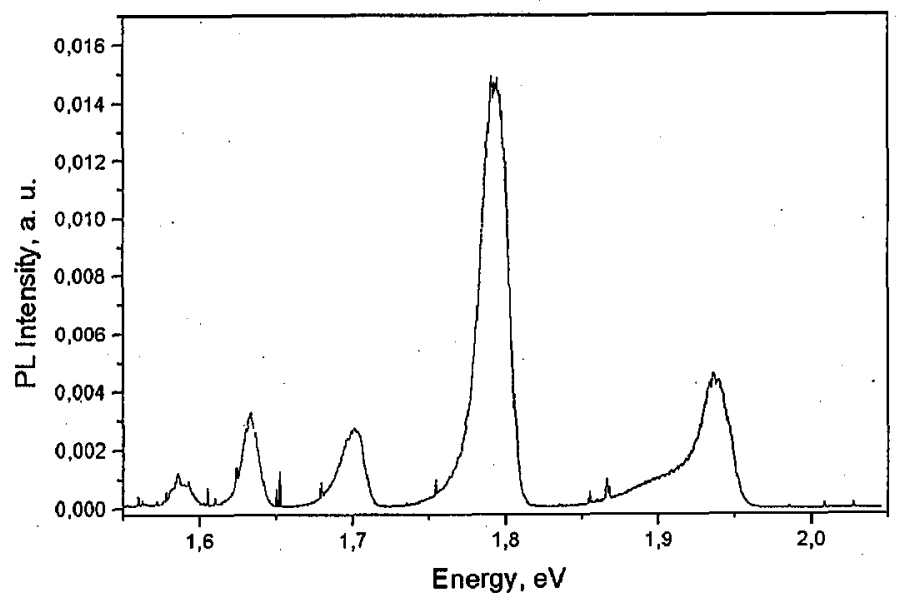

Fig. 2. Photoluminescence spectrum of multiquantum well on a hybrid substrate $\mathrm{CdTe} / \mathrm{GaAs}$ cleaved along $\langle 110\rangle$ direction (there are 24,12 , and $6 \mathrm{ML}$ wide quantum wells embedded in CdMnTe barriers). 
grown buffer layer present in the samples described above. Since this particular layer has a smoothing effect, the growing of the CdTe QW directly on the cleaved surface, as required by the T-shaped wire structure design, will lead to further deterioration of the optical quality.

This work was partially supported by the Committee for Scientific Research under grant PBZ 028-11.

\section{References}

[1] A.R. Goni, L.N. Pfeiffer, K.W. West, A. Pinczuk, H.U. Baranger, H.L. Stromer, . Appl. Phys.Lett. 61, 1956 (1992).

[2] L. Pfeiffer, K.W. West, H.L. Stromer, J.P. Eistenstein, K.W. Baldwin, D. Gershoni, J. Spector, Appl. Phys. Lett. 56, 1697 (1990).

[3] H. Mariette, D. Brinkmann, G. Fishman, C. Gourgon, Le Si Dang, A. Löffler, J. Cryst. Growth 54, 1872 (1996).

[4] G. Cywiński, T. Wojtowicz, G. Karczewski, J. Kossut, W. Gębicki, Acta Phys. Pol. A 94, 277 (1998). 